\title{
Sugar-like gravel in the gearbox and the question whether diabetes is a coronary artery disease equivalent
}

\author{
Michael J. Zellweger, $M D^{a}$ \\ a Cardiology Department, University Hospital Basel, University of Basel, Basel, Switzerland
}

Received Feb 5, 2021; accepted Feb 5, 2021

doi: $10.1007 / \mathrm{s} 12350-021-02577-y$

See related article, pp. 1222-1233

With the broader availability of Positron Emission Tomography (PET) myocardial flow measurements at rest and during vasodilator stress became reality in routine non-invasive cardiac imaging. Assessment of myocardial blood flow improved diagnosis and risk assessment in the evaluation of patients undergoing perfusion PET imaging for coronary artery disease evaluation.

Large studies have reported on the incremental value of coronary vasodilator assessment over perfusion data, above and beyond the assessment of myocardial ischemia and scar. Even in patients with neither scar nor ischemia, myocardial blood flow measures provided incremental prognostic value. ${ }^{1-3}$ In patients with normal qualitative scan results, the cardiac death rate was 36 times higher in patients in the lowest tertile of myocardial flow reserve (MFR) compared to the highest tertile, $3.6 \%$ and $0.1 \%$, respectively. ${ }^{1}$ Moreover, the same authors demonstrated that coronary vasodilator dysfunction is a powerful, independent predictor of cardiac mortality in both, diabetic and non-diabetic patients. $^{3}$

Decades ago, the controversy started, whether diabetes should be considered as a CAD equivalent ${ }^{4}$ : Over a follow-up period of almost 8 years, Haffner et al evaluated four groups of patients: patients without diabetes and no prior myocardial infarction, patients with

Reprint requests: Michael J. Zellweger, MD, Cardiology Department, University Hospital Basel, University of Basel, Petersgraben 4, 4031 Basel, Switzerland; michael.zellweger@usb.ch

J Nucl Cardiol 2021;28:1234-5.

1071-3581/\$34.00

Copyright (C) 2021 American Society of Nuclear Cardiology. diabetes but no prior infarction, patients without diabetes but prior myocardial infarction, and patients with diabetes and prior myocardial infarction. ${ }^{4}$ The outcome data demonstrated that diabetic patients with prior myocardial infarction had the highest mortality, whereas nondiabetic subjects without prior myocardial infarction had the best prognosis. Diabetic patients without prior myocardial infarction and nondiabetic patients with prior infarction had similar outcomes. ${ }^{4}$

Irrespective of the fact, if one calls diabetes a CAD equivalent, the disease is linked to high cardio-vascular risk, morbidity and mortality.

According to the most recent standards of care by the American Diabetes Association, it is recommended that patients with diabetes aged 40 to 75 years even without atherosclerotic cardiovascular disease use moderate-intensity statin therapy in addition to lifestyle modification. $^{5}$

Even Aspirin therapy (75 to $162 \mathrm{mg} /$ day) may be considered as a primary prevention strategy in diabetic patients who are at increased cardiovascular risk, after a comprehensive discussion with the patient on the benefits versus the comparable increased risk of bleeding. ${ }^{5}$

Thus, diabetic patients definitely are at increased cardiovascular risk and therefore need patient-tailored care and treatment.

In this issue of the Journal, Assante et al evaluated the interrelation between myocardial blood flow and cardiac events in a matched patient population of diabetic $(n=451)$ and non-diabetic patients $(n=451)$ with suspected CAD and normal qualitative myocardial perfusion as assessed by Rubidium $\mathrm{PET}^{6}$.

The main findings were that MFR was lower in diabetic patients than in non-diabetic patients, before and after matching. In patients with lower MFR the outcome was worse.

One of the question is, why-what are the most important factors leading to a lower MFR — and can they be modified? The authors mention diabetes, age and 
hypertension as independent predictors of reduced MFR (in the matched cohort). It might have been interesting to look also at the non-matched population here. In addition, information about duration of diabetes, endorgan damage (e.g. diabetic retinopathy, microalbuminuria, (autonomic cardiac) neuropathy, and peripheral or carotid occlusive disease) would be important variables to incorporate into further studies. Since knowing the predictors of a reduced MFR in diabetes (which is probably also a surrogate marker for the presence of a metabolic syndrome) may result in more understanding of its mechanisms and may lead to new treatment targets?

The outcome results of the study ${ }^{6}$ are in line with the published studies in the literature and underscore the complimentary value of perfusion and flow information: Diabetic patients with reduced MFR had a higher event rate than those with preserved MFR and nondiabetic patients with preserved or reduced MFR. The event rate was similar in patients with diabetes and normal MFR and patients without diabetes but reduced MFR. Principally, these findings are in line with Haffner's study mentioned above ${ }^{4}$ (with the endpoint of death in the latter) and the "diabetes $=\mathrm{CAD}$ equivalent" theory, even though the overall event rate in the study by Assante et al was very low $(n=33)$. In addition, a composite endpoint of cardiac death $(n=10)$, non-fatal myocardial infarction $(n=12)$ and unstable angina requiring coronary revascularization $(n=11)$ was used. Keeping these low event rates in mind, one might ask how robust the results of the subsequent analyses are? Also the fact, that there was no independent predictor of events in the non-diabetic group, is probably due to the very low event rate in this particular sub-group?

The authors underscore the potential and importance of MFR measurements as a risk stratification tool in (diabetic) patients. Certainly, low MFR is consistent with higher mortality rates, but we do not know, if more intensive, preventive efforts lead to better MFR and thus to lower mortality? Furthermore, it is not known if the most accurate treated diabetic patients have the highest MFR?

However, in summary, the study by Assante et al adds evidence to the "diabetes $=\mathrm{CAD}$ equivalent" hypothesis and underscores the importance that diabetic patients are treated accordingly as high risk cardiovascular patients and as suggested by the guidelines and recommendations.

\section{Disclosures}

There is no conflict of interest.

\section{References}

1. Murthy VL, Naya M, Foster CR, Hainer J, Gaber M, Di Carli G, et al. Improved cardiac risk assessment with noninvasive measures of coronary flow reserve. Circulation 2011;124:2215-24.

2. Herzog BA, Husmann L, Valenta I, Gaemperli O, Siegrist PT, Tay $\mathrm{FM}$, et al. Long-term prognostic value of $13 \mathrm{~N}$-ammonia myocardial perfusion positron emission tomography added value of coronary flow reserve. J Am Coll Cardiol 2009;54:150-6.

3. Murthy VL, Naya M, Foster CR, Gaber M, Hainer J, Klein J, et al. Association between coronary vascular dysfunction and cardiac mortality in patients with and without diabetes mellitus. Circulation 2012;126:1858-68

4. Haffner SM, Lehto S, Rönnemaa T, Pyörälä K, Laakso M. Mortality from coronary heart disease in subjects with type 2 diabetes and in nondiabetic subjects with and without prior myocardial infarction. N Engl J Med 1998;339:229-34.

5. Addendum. 10. Cardiovascular Disease and Risk Management: Standards of Medical Care in Diabetes-2020. Diabetes Care 2020;43: S111-S134. Diabetes Care 2020;43:1977-8.

6. Assante et al. J Nucl Cardiology.

Publisher's Note Springer Nature remains neutral with regard to jurisdictional claims in published maps and institutional affiliations. 